\title{
The sexual use of a social networking site: The case of Pup Twitter
}

\begin{abstract}
This article examines how Twitter has been adopted and used by a sexual subculture in distinct ways. Drawing on interviews with 26 gay and bisexual men based in the UK who identify as 'pups', it demonstrates how a kinky sexual subculture exists on a social networking site in new and innovative ways, adapting various elements of Twitter to form a unique subculture that I call 'Pup Twitter'. Engaging with debates about social trends related to sexuality, as well as contemporary understandings of social networking sites, the study documents how this subcultural sexual community, while predating Twitter, has adopted online methods to enhance communication, engagement and even visibility. The intersection of sexuality and social networking sites is an area ripe for further study, and this article develops empirical and conceptual ways to examine this issue in the future.
\end{abstract}

KEYWORDS: bdsm; community; kink; online; sexuality; Twitter. 


\section{Introduction}

The rise of social networking sites (SNS) in society is part of a significant and prolonged trend of changing internet technologies. Globally, SNS are entrenched in people's lives, with the two main SNS in Western society being Facebook and Twitter. While research has more strongly highlighted the role of Facebook in helping form online communities, this article focuses on the use of Twitter in developing a sexual subculture for people who participate in 'pup play’ (Wignall and McCormack 2017). Pup play is a kinky, often sexual, activity where individuals imitate the posture of a dog and wear a collar and other dog-related items. Pup play can also be a social activity, a tool for relaxation, and it mirrors other forms of play.

Focussing on the case of pup play, this study addresses the absence of knowledge around how sexual communities use SNS. Analysing in-depth interviews with 26 young gay and bisexual men who engage in pup play and have a profile on Twitter that discusses pup play, I provide evidence of how Twitter is being adopted and adapted by the pup community: helping to foster community online, while simultaneously facilitating a related offline community. This use of Twitter, which I call 'Pup Twitter', is used by participants as a tool for communication and visibility. As such, I develop knowledge and advance sociological debates around the intersection of new media, communities and sexuality in contemporary times.

\section{SNS and Online Social Communities}

Early online interactions occurred on discussion lists or bulletin board systems. As technology developed, these online interactions developed into more meaningful forms of communication where friendships could develop as well as a sense of community (Smith and Kollock 1999). Forums and chat rooms were particularly useful for the development of niche communities, including sexual minorities. For example, Wakeford (2002) discusses 
cyberqueers who used the internet to explore their identity, engage with queer spaces and meet other queers.

The development of Web 2.0 led to the SNS platform we are more familiar with today, such as Facebook and Twitter. Central to this was the way that communication moved from being asynchronous, where replies could occur months after the initial message, to synchronous, where replies could be near-instantaneous in a conversational manner. Contemporary popular SNS combine synchronous and asynchronous forms of interaction. Boyd and Ellison (2007: 211) define SNS as:

A web-based service that allows individuals to (1) construct a public or semi-private profile within a bounded system, (2) articulate a list of other users with whom they share a connection, and (3) view and transverse their list of connections and those made by others within the system.

A key feature within this framework is that SNS revolve around interactions occurring between two or more people - often leading to the formation of online discussion groups or communities (Preece and Maloney-Krichmar 2005).

SNS are integrated into everyday social practices, to the extent that they have become almost a ritual performance of the self (Rettberg 2014). Yet this performance of the self, while disembodied, is increasingly recognized as being located within various online communities. Indeed, the term 'community' has become increasingly important among users of SNS (Baym 2015). Initially, definitions of online communities focused on group displays of camaraderie, empathy and support online (e.g. Smith and Kollock 1999). Others highlighted the more instrumental purpose where individuals are brought together with a shared purpose, online (e.g. De Souza and Preece 2004). The debates around what constitutes an online community echo those concerning how an offline community is understood (Chayko 2008). 
Baym (2015) identities five qualities of community related to online communication: a sense of space; shared practice; shared resources and support; shared identities; and interpersonal relationships. These five qualities are valuable, and provide useful detail to the more open understanding of online communities by Preece and Maloney-Krichmar (2006: 00), who argue that 'a more productive approach may be to accept [online] community as a concept with fuzzy boundaries that is perhaps more appropriately defined by its membership'.

Contrary to early findings suggesting detrimental effects with engaging in online communities (e.g. Kraut et al. 1998), recent research has highlighted the benefits of such engagement: that real-world social networks use online platforms to communicate with each other (Boase and Wellman 2006); SNS can increase social capital, and improve self-esteem and increasing life satisfaction (Ellison et al. 2007); virtual platforms can provide a support network in medical settings (Moorhead et al. 2013). Indeed, Baym (2007) highlights how SNS support online communities by 'providing launching pads for individuals to connect with one another. People may build personal relationships. These go beyond simple friending to include sending one another personal messages that lead to other kinds of interpersonal contact'. Despite the discourse around the plethora of the online for different communities, there are still communities which have a dearth of research - specifically sexual communities.

\section{Offline Sexual Communities}

Examples of sexual communities are varied and can range from the more widely known and established Lesbian, Gay and Bisexual (LGB) communities (Ghaziani 2014), to more subcultural sexual communities, such as those organized around specific sexual kinks (Rubin 1991). 
Early sexual communities formed as a response to the stigma towards sexual minorities and because of legal discriminations. The death penalty was used as punishment for 'buggery' - which was defined as sex between two men (Peakman 2013). While the death penalty was lifted in 1861, homosexuality was still illegal in the UK until 1967 and classed as a mental disorder until 1973 in the Diagnostic and Statistical Manual (Drescher 2015). The legal, cultural and psychological framings of homosexuality had great consequences on gay subcultures through the fear of criminal proceedings and stigma, meaning that they were forced underground across much of this time.

As a response, gay men would meet to socialise and have sex in subcultural venues called Molly Houses (Norton 1992). A later iteration of gay subcultural venues were gay bars and clubs, where people would meet for community, alcohol consumption and sexual liaison. Ghaziani (2014) calls these areas homogenous areas 'gayborhoods', which not only provide areas of safety for sexual minorities, but also help to challenge heteronormativity.

These sexual communities sought to reduce the amount of stigma individuals' received, even if this meant distancing themselves from other sexual minorities. This was partly as the result of the gay rights movement which consolidated around certain 'legitimate' sexual identities (Weeks 2007). Rubin (1981: 117) highlights how the lesbian and gay communities stigmatized both heterosexual and non-heterosexual kink communities, 'hastening to disassociate themselves from [kink communities] without challenging the distorted picture of [kink] itself'. In doing so, LGB communities contrasted themselves with something deemed morally worse, shifting stigma towards kink communities. While this helped LGB people to make gains toward equality in society, it could be argued that it was to the detriment of kink communities.

Rubin (1991) undertook one of the earliest ethnographies of a kink community. She documented the San Francisco Catacombs between 1975 and 1981, a place known for its 
leather and fisting parties. She discussed the caution involved in becoming part of this community and the steps involved in attending one of these parties. Most, if not all, of the interactions occurred in person and there were several vetting procedures for access to the club. She wrote:

It was not easy to get into the Catacombs... To be invited to the parties, you had to be on Steve's list. To get on Steve's list, you had to be recommended by someone he knew, and often had to be interviewed by him as well. (1991: 227).

Within this context, kink communities remained very much as subcultural groups, hidden by a dominant gay culture that ignored much of its historical association with kink and leather.

However, changes in cultural norms and increasing tolerance towards sexual minorities has led to a shift in gay sexual cultures. Ghaziani (2014) highlights how gayborhoods are rapidly changing, with less need for closely located geographical spaces to create lesbian and gay 'scenes'. This disintegration of hegemonic gay scenes has led to the proliferation of other diverse forms of sexual minority communities. He argues that we live in a world 'not of shrivelling sexual and spatial expressions but instead of extraordinary growth and new possibilities' (Ghaziani 2014: 259). While Ghaziani focuses on diversification in terms of geography and type of community establishment, the plurality can be seen in the increased visibility and recognition of a range of communities organised around other sexual practices_-including group sex (Frank 2013), furries (Soh and Cantor 2015) and pup play (Wignall and McCormack 2017). These communities, while predating the internet, have adopted online methods to enhance communication, engagement and even visibility.

\section{Online Sexual Communities}

Technology has had a major impact on sexual communities. Döring (2009) highlights that the use of the Internet is a key component in the lives of sexual minorities today. He states: 
By providing an easily accessible platform for the establishment of contacts between individuals of similar creeds and sexual orientations, the Internet can ameliorate social isolation, facilitate social networking, strengthen self-acceptance and self-identity, help to communicate practical information, and encourage political activism, among other things.

Döring (2009) sees these features as highly influential for sexual minorities in developing sexuality-oriented communities. Reflecting this, there are examples of websites which serve a purpose of allowing sexual minorities to communicate (e.g. Gray 2009). While many of these focus on the social, there are numerous examples which focus on the sexual and specifically hooking up (e.g. Gudelunas 2012; Blackwell et al 2014).

Yet the classification of websites into either sexual or social as discrete and exclusive categories is unrepresentative of how users engage with the sites. For example, Mowlabocus (2010) highlights how Gaydar, a profile-based dating website for men seeking men, serves a dual purpose of allowing for social communication and friendship ties as well as allowing for dating and casual sex. Similarly, Blackwell et al. (2014) discuss how Grindr, a location-based real time 'dating' app, was originally a phone application used to seek immediate sex with other men seeking men, but has also been used for socialising and making friends. While some individuals manage their profiles online to limit and manage their sexual disclosure (Jaspal 2016), this is markedly different to the exploration of fantasy in wholly anonymous online spaces (Waskul 2003). Rather than focusing on categorising such websites as serving a sexual or social function, I label them socio-sexual networking sites (SSNS) due to the dual nature of allowing for communication alongside the opportunity for sexual encounters. In this way, they can be considered an online gay scene, serving the dual facility that gay bars and clubs have historically done (Ghaziani 2014). 
While there is a growing body of research that investigates the dynamics of SSNS for sexual minorities, my research into a sexual subculture found that Twitter used to develop community and make connections, rather than SSNS being used (Wignall and McCormack 2017). Yet virtually no research examines this issue: the one study that does examines female porn consumers use of Tumblr to share porn clips and experiences (Mondin 2017)—a markedly different SNS and a demographic that does not have a related off-line community. While there has been an expansion of research on kink communities (e.g. Newmahr 2011; Weiss 2011), these have not examined the role of the online in communities.

Twitter, a microblogging service, started out as a way to 'follow' celebrities and politicians, giving short updates on daily activities, providing a place to microblog or keeping up to date with popular trends (Honeycutt and Herring 2009). However, it has developed into a forum for global conversations on all topics. For example, Twitter is being used to transmit breaking news before traditional media and plays a role in online political discussions (Hu et al., 2012) and it creates a 'virtual loungeroom', connecting the active audiences of specific TV shows at an unprecedented scale' (Harrington et al. 2012). Highlighting this, Murthy (2012: 1061) suggests Twitter should be seen as a social media site because of its design to "facilitate social interaction, the sharing of digital media, and collaboration", normally with strangers rather than creating online communities (Murthy 2010).

While media consumption and global conversations were the intended functions of Twitter, research has discussed how new media are being re-appropriated in different ways by its members (David 2010). Indeed, the functions of Twitter are vast and have been adapted by users in several ways. Newmahr (2015: 63) succinctly summarises the impact of Twitter, commenting:

Facebook and Twitter have impacted everyday life on multiple levels. These technologies have changed how we feel our moments and what meanings we make of 
them, our definitions and frames for social interaction and approval, our conceptualisations of the private/public, and the intersections between privacy and intimacy.

There is increasing focus on Twitter and the formation of online communities in research. Gruzd et al. (2011: 1313) state, 'Twitter is a good case to understand how people integrate information and communication technologies (ICTs) to form new social connections or maintain existing ones.'

\section{Aims and Objectives}

This study examines the use of Twitter by a sexual subculture - those who engage in pup play. In particular, the function of the online space was examined, as well as why participants' used it over established kink-orientated SSNS which offered similar features. By examining the narratives of participants who identify as part of the pup community and have active online Twitter profiles, I begin to breakdown the online space of this sexual subculture and situate the discussion in broader sociological debates.

\section{Methods}

Participants are part of a broader study on individuals who engage in pup play. Pup play is an emergent socio-sexual behaviour and has been described as a kinky sexual activity, where individuals adopt a submissive role, imitate the posture of a dog, and wear "gear" associated with owning a dog (Wignall and McCormack 2017). While the sexual aspect is recognized as an important component for most 'pups', there is also an emphasis on sociality and community - including regular social events, the development of friendship circles, and networks of 'pups' in various countries (Vice 2015). Those who engage in pup play recognise it as kinky, but on the "lighter end of the spectrum" and different to other kink activities 
(Wignall and McCormack 2017), although there are significant geographical and cultural differences in how it is experienced.

\section{Participants}

Data comes from 26 gay and bisexual Caucasian men who engage in pup play. The sample is limited to gay and bisexual men due to the exploratory nature of the study. Participants were aged 18-35 $($ mean $=23.73$, median $=23)$ at the time of interview and from working and middle class backgrounds, based in towns and cities across the UK.

Given the dearth of prior research, a qualitative approach was employed to study explanations, narratives and meanings behind pup play (see Rehor 2015). A symbolic interactionist approach was adopted because it privileges individuals' narratives in understanding a phenomenon, recognising that the meaning of events is situated within human interactions, the context of these dynamics and how they are interpreted by those involved (Plummer 1995).

\section{Procedure}

Participants were recruited through various SNS, primarily Twitter. The author made contact with accounts which featured pup play related information, asking if they would participate in academic research on pup play. Additional information was given to those who responded positively. Participants decided where the interview took place, with most opting for a café or a university environment. Six interviews were conducted over Skype.

Semi-structured in-depth interviews were employed, lasting approximately 60 minutes and were carried out between December 2014 and April 2015. Interviews focussed on: what pup play is; how participants engaged in it; how participants became involved in it; and use of SNS and SSNS in relation to pup play. Due to the nature of semi-structured 
interviews, participants were not asked precisely the same questions, and the interviewer asked a number of follow up questions. All interviews were digitally recorded and transcribed.

The data was analysed using a modified grounded theory approach (Charmaz 2014). Emerging codes were developed into focused codes. Undertaking a form of analysis called middle-range coding (Dey 1993), analysis continued in conjunction with a more focused search of the literature. As such, inductive themes were combined with existing frameworks to develop a theory of the phenomena grounded in the data and engaged with existing literature (Urquhart 2013). Following this, an independent researcher cross-checked the analysis with five randomly selected transcripts to check internal coherence. It is through the processes of coding, logical abstraction and inter-rater reliability that rigor is assured.

Ethical approval was granted, and interviews conducted in accordance with British Sociological Association ethical guidelines: all participants read a participant information sheet, and signed a consent form prior to the interview. To ensure anonymity, pseudonyms are used in this article. Given the intimacy of the pup community, participants may be identifiable to other participants through the quotes. To address this issue, some minor details in quotes may have been changed to preserve anonymity.

\section{Pup Twitter: Reasons and Usages}

While all participants discussed using SNS more broadly, the use of Twitter was widespread among participants, and it is the central internet mechanism by which they communicated with each other. Participants also regularly used Facebook, which was normally for their nonpup identity; Tumblr, which some participants used for their pup identity while others used it more generally; and Fetlife, a SSNS for those interested in kink, but this was a small number of participants. 
Participants gave several reasons for creating a Twitter account. Primarily, they were introduced by somebody who already had one. For example, Warren said, 'Another pup got me onto Twitter. He already had an account and whinged at me until I got one. Now I tend to whinge at other pups until they get one.' Similarly, Neil said, 'I've had a pup friend tell me "you need a kinky pup Twitter" over and over again. I eventually got to the point of defeat and created one.' Allan summarises the community pressure to join Twitter, saying, 'I got Twitter by popular demand, it wasn't my choice!' Sam explained why he told other pups to join Twitter, saying, 'That's how we do it now. They join Twitter because we tell them it's how they can meet people. You don't have to, but it's the easiest.'

Those not directly told to join still recognised that Twitter featured as a central role for the pup community. For example, when asked why he joined Twitter, Richard said, 'It seemed like everybody was on it. At a house party for pups, I met new people and $80 \%$ of them asked me for my Twitter. I wanted to keep in touch with them, so I created a Twitter account.' Bruce said, 'When I was searching online for general kink things, I came across pup play. When I carried on searching for pup stuff, a lot of the sources were Twitter.' Similarly, Tom said, 'I was looking at other things on Tumblr [a blogging site] and came across some pup stuff from Twitter - I eventually got one myself.'

The way Twitter was primarily used by the participants reflected its utilization in society more broadly - a microblogging platform allowing for simultaneous communication with multiple people, the ability to share media, and to follow the updates of other users (Murthy 2012). Furthermore, given the public nature of Twitter, it allowed participants to easily search for other individuals with similar interests and witness conversations between public members.

When asked how he used Twitter, Dexter said, 'It's a great tool for communicating and making friends... Seeing everyone on Twitter being available to help you or offer advice 
is great.' Similarly, Bart said, 'I use it to interact with other pups, cyber role play, and general chats. Twitter is the primarily exploration of pup play for me.' John discussed how his use of Twitter changed the more he used it, saying, 'At first I would occasionally post pictures, but I hardly used it... I started speaking to more people and it gradually increased. I've now got over 1,000 followers and it's more a social thing now.' Twitter enabled the exploration of fantasy in a safe environment, providing the option to engage with others while not venturing into physical spaces and kink venues that are more overtly sexualized and stigmatized in wider culture (Newmahr 2011).

While the use of hashtags serves a role within Twitter more broadly to allow individuals to contribute to national and international conversations, their function was not the same for participants. Hashtags were generally not used, as participants were not seeking to interact with individuals outside of their community. However, hashtags served a functional purpose when participants were attending offline events - hashtags allowed a conversation to occur around the event. As Bruce explained, 'I used the hashtag \#puppypride to find out who was going to the event so I could chat and get to know people beforehand.' This highlights both the role of Twitter in facilitating offline community events, and also how it is a subcultural element of Twitter through the participants avoiding more general hashtags.

An analysis of the participants' profiles demonstrated the main ways that Twitter was used. These were: to Tweet about past or future sexual encounters; to post explicit sexual images; to communicate with other members (both sexually and non-sexually); and to post life updates (e.g. work related information, life events, general life updates). The mixture of posts helped to contribute to the sense of community for participants.

Indeed, the use of Twitter can be seen as a distinct usage of Twitter by 'pups' as there are distinctive characteristics of what I call 'Pup Twitter'. First, all but one of the name/handles consist of the word 'pup' either following or followed by the name they ascribe 
to themselves as a pup, that is '@PupPseudonym'. Secondly, images of the Twitter profile pictures tend to relate to pup play - 20 of the 26 participants had a picture of themselves wearing a pup-like mask, normally in a dog-like posture. Thirdly, most participants had some reference to pup play in their bio (biography) sections on their Twitter profiles, such as a description on what type of pup they are or references to other pups they know. Finally, the majority of the communication tended to be aimed at other pups; indeed, the overall impression given by participants was that the online pup community, while being welcoming to new members, was homogenous. These four characteristics are a distinct usage of 'Pup Twitter' that are not reflected on the SNS more broadly, supporting the framing of an online community.

All participants who were on Twitter were also on SSNS, such as Recon or Grindr. It was common on these SSNS to see participants' Twitter handles mentioned either in an 'about me' section or reflexive in their profile name. For example, a Twitter handle maybe ‘@pupPseudonym' while on the SSNS the profile name would be 'Pup Pseudonym'. Not only did this provide a way of recognition for finding friends and across other platforms, it also emphasises the online identity - participants generally only had one profile deemed Not Safe For Work (NSFW). NSFW is "an acronym used widely on social media to annotate content that you would not want on your screen, should your colleagues see over your shoulder" (Tiidenberg 2016: 1564). Moreover, Twitter allowed for the movement from the SSNS to the more community/personal pup Twitter account.

\section{Negotiating Privacy on a Public SNS}

Ten of the 26 participants ensured their Twitter profile was private - this meant that in order to see the posts from the private account, an individual must first have a Twitter account and send a request to follow the individual. When asked how he managed his Twitter profile, 
Luke said, 'I keep my profile private and generally keep an eye on the photos of me getting around.' Similarly, Sam said, 'I lock my account so you can accept people who follow you. That's the ultimate sanction.' Warren said, 'My Twitter profile is protected so I only accept people that are into kink. When somebody clicks to follow me, I see who they are and check out their profile to see if it's active and if they're into kink.' When asked why he protected his account, Leon said, 'Having a protected account is so freeing. It's like having a private bit of the internet where I can social network with people and do it in my own way.' He added, 'I don't want my family finding out about my kink friends - they're very old fashioned.'

While privatising a Twitter account may control for unwanted audiences, participants also spoke of how it can reduce the number of followers received of the target audience. For example, Jason said, 'My profile is locked, which puts a lot of people off straight away. But it makes it so that if people really want to follow me then they will ask to.' Dexter said, 'I have gone through stages. I started anonymously and public and amassed over 1,000 followers. I freaked out then blocked the majority of people I didn't know and converted my profile to private where I had more control.' Richard said, 'I have a lot of followers, about 2,000 now. I did lock my account for a bit, but then it reduced the activity I could do with new pups, so I unlocked it again.'

Protecting an account was not the only way participants managed their privacy and identity. Leon discussed how he took elaborate measures to prevent being discovered, saying: I have my face on there, but I purposefully used a different email address from my regular one. I use a different login name and I use different face pics from the ones on Facebook. So you can't google it and without the name you can't find your way in. I don't think it's searchable, or indexed.

Focusing more on monitoring who follows him, Warren said: 
I tend not to add people that I don't already know. I don't just randomly go through Twitter and add everybody with pup in their profile - normally I will follow a new person if I'm told about them by somebody else, or if I end up having a conversation with them, or if I know they're in a relationship with somebody I already know.

While determining who could follow their accounts was an important issue for some, participants also discussed the methods involved in choosing who they should follow. For some, they followed accounts that had pup play related information. For example, Chase said, 'I tend to follow people with stuff on their profile similar to mine.' Luke reflected this view, saying, 'I added some pups and furries on Twitter because they interested me. I also started to discerning pups on Twitter from their online videos on other sites.' Ben discussed, while also following other pups, how he followed social media accounts of related companies, saying, 'I followed the main pups on Twitter... The Dogs Bollox and a few pup companies on there too are good to follow to see information and pictures from events.' However, some participants were more restrictive over who they followed. For example, Warren said, 'I don't just follow anybody - I tend to meet them at an event or be introduced by somebody else.' Similarly Bruce said, 'I only follow people who I sort of know or I'd have a very full Twitter feed. Ain't nobody got time for that!'

Participants also discussed how they were wary about self-disclosure on Twitter. For example, Steven discussed editing pictures before posting them online, saying, 'I have posted pictures in which you can see my full face, but I normally blur them out.' Richard said, 'I don't post many pictures with my face in them - when it has my face in it, they're never XXX. If I am getting serially retweeted by an account I block them.' Jimmy said, 'Given my job, I need to be careful what I post. I don't post face pictures or real information about me I'm known by my Twitter name.' While the withholding of face pictures was the primary 
method of managing their profiles, restricting "real-world" information (e.g. real names, jobs, non-kink SNS) was also used to limit self-disclosure.

Promoting the idea of self-disclosure on their pup Twitter profiles, some participants indicated that they had another Twitter account; normally this other account did no0t mention their pup identity, was non-kink orientated and more reflexive of their 'non-pup' lifestyle.

For example, Bruce said, 'I have two accounts: a pup one and a non-pup one... I use the nonpup one for work based things and it's very much non-kinky.' Similarly, Carl said, 'I already had one Twitter account, but I didn't want to blur my kink and vanilla life too much, so that's the driving reason behind my kinky/pup Twitter. It was a way of keeping them both separate.'

\section{Evaluating Pup Twitter}

While primarily a microblogging platform, Twitter has helped form an online community for this sexual subculture. Despite other SNS already existing which are more orientated towards creating a community (e.g. Facebook), and SSNS which are orientated more towards sexual subcultures (e.g. Fetlife, Recon), Twitter was the preferred means of interacting online with others from the same sexual subculture for 24 of the 26 participants. Participants spoke of both positives and negatives when engaging with this online community.

When asked why Twitter was the preferred website, responses were oriented around the characteristics of Twitter. For example, Sam said, 'I have met more pups on Twitter than any other website. I think the reason behind it is the character limit - you can't over express yourself in a way and constrict yourself. Tweeting is very easy to do.' Highlighting the combination of synchronous and asynchronous communication, Andrew said, 'Twitter is excellent. You and 1000 other pups can all talk in pup terms, share pictures of what you do, organise meets. Other sites don't have that interactive edge, you have the feed and it's easy to 
navigate.' Similarly John said, 'You can explore a lot more on Twitter, most people have it, you can see what other people are saying and that's how people get more involved.' Jason also highlighted the multi-conversational aspect of Twitter, saying, 'Twitter isn't forum based, you can still message people in private like other sites, but you can have group conversations and stuff much easier on Twitter, It's instantaneous and you can have conversations with several people all at once.' While there is evidence of exclusion within sexual communities by its members (e.g. Callander, Holt and Newman 2015), as well as harassment and hate speech, these issues were absent from participant narratives.

The popularity of Twitter more broadly as an SNS has resulted in the development of mobile applications, with the intention of easily integrating Twitter into a daily life. This was highlighted by participants as preference for its use. For example, Steven said, 'I have Twitter on my phone so it's just easier to use.' Highlighting Twitter's facility to switch easily between different accounts, John said, 'Most people will use Twitter. You can use it on your phone as well. By the flip of a switch on Twitter, I can change between normal or kinky name.' Similarly, Greg said, 'I'm on the other sites, but I tend to use Twitter more. You can have the app on your phone, it doesn't get blocked anywhere when using it as it's not inherently sexual.'

However, there were some worries about having multiple Twitter accounts on the same device. For example, while Steven praised the ease of having Twitter on a mobile device, he said:

I make sure I post different things on my normal and my pup Twitter profiles. I have cross posted before though. A sexual scene aimed at my pup account, but posted it from my (normal) account. I was on the underground and didn't realise - it was only 6 hours later that I decided to check my accounts and realized. I think I lost about 4 followers. It was vanilla porn so it wasn't too bad, but it could have been much worse. 
Steven was not alone in accidentally cross posting. Warren said, 'I once did it a while ago, but deleted it quickly... It's a risk, but I've worked out how to avoid it.' Similarly, Dexter said, 'I cross posted a few times, so now I don't have access to both accounts on the same phone.' While these cross-postings luckily had no negative consequences, it is easy to see how the circumstances could have been worse.

Cross-posting was not the only concern raised about having a Twitter account. Participants discussed the possibility of their Twitter account being discovered by their family or non-kink friends. Chris spoke about the negative consequences he faced when friends found his profile. He said:

My profile was discovered by my church friends - I live with some of them and they're not the most understanding. They suspected I was up to stuff and went searching. She found my Twitter account and said I either shut it down or move out the house. That was about 6 weeks ago and it's been shut down... I never thought about the consequences of it. For me, it was a separate life, so I never thought it would be a problem.

While Chris was not alone in having his Twitter profile discovered by friends or family, he was the only one he had negative ramifications.

Some participants had no significant concerns. For example, Freddie said, 'It doesn't bother me; I'm not embarrassed by it. Is it really an issue?' Similarly, Jamie said, 'I know people worry about that sort of thing, but I think if my family see the profile, then it's because they've been searching for it. If they search gay fetish pups, they're going to find me' When asked if he done anything to maintain privacy on the account, Andrew replied, 'No not really. I have pictures of where I live, my location is accurate, sometimes I post the same images on all my sites and my pictures have my face in them.' 
Participants employed techniques to minimize the likelihood that their profiles could be discovered - some closely monitored then, while others took a more lax approach. The extent to which participants monitored their profiles was influenced by several factors, including participants' professional employment, their knowledge of Twitter privacy tools and the openness of their kink identity to the non-kink community.

\section{Discussion}

This article has drawn on 26 in-depth interviews with gay and bisexual men who participate in pup play and use a Twitter account as an extension and exploration of their pup identity, documenting the emergence of 'Pup Twitter'. It examines why participants create a Pup Twitter account; what they use it for; the potential benefits of engaging with it; and possible negatives of having a profile. Most importantly, the article documents the ways in which a traditional SNS is being used to interact and develop community amongst a sexual subculture, rather than using existing SSNS.

Reasons for the use of and preference for Twitter by participants, rather than other SNS or SSNS, centred on the features of the website. This included the possibilities for both synchronous and asynchronous communication, the flexibility on levels of privacy, the use of pseudonyms, and the ability to easily search for and follow other 'pups'. Yet the participants also adapted these features in specific ways: the distinct use of profile pictures and names separated participants from other users, and a subcultural community was formed online. Unlike other SNS, such as Facebook, Twitter allows the creation of multiple profiles and allows users to switch between profiles easily. This plurality of Twitter profiles was deemed vital for participants in that it allowed for both separation and closeness between different aspects of their overall 'online identity.' This raises interesting areas for further investigation, 
including the ways in which these separate profiles differ and how they relate to the individuals' broader identity and social life.

The use of Twitter to serve a more specialised purpose is not unique to the participants in this study. Indeed, an ongoing debate in the sociology of new media centres on whether communities are structured around available technologies or if communities adapt technologies to suit their purpose. David (2010: 20) argues for a middle ground:

...the Internet has effects. Interactions that would not otherwise occur, do occur. Yet the medium itself does not determine how people will interact. The way that the Internet is used itself is a performance, the medium is interpreted and applied in ways determined by social negotiation between the parties to the interaction.

Hines (2010: 116) develops a similar argument within a poststructural frame, contending that the space created for interaction on the Internet is both performative and a performance, meaning that it both structures communities but these communities also perform in particular ways for the SNS.

This research supports this perspective. Participants in this study complicate the notion of Twitter being classified as either a social networking site or a social media site (Murthy 2012). There are narratives of media consumption and interactions with strangers and popular kink profiles reminiscent of social media technologies (e.g. Murthy 2010), alongside narratives of using Twitter to continue interactions which occur offline, more reflexive of social networking sites (boyd 2007; Ellison et al. 2007). Additionally, this study also suggests that arguments that SNS and new media technologies promote isolation and loneliness (e.g. Turkle 2011) do not take account of the range of ways SNS can promote positive human interaction.

Twitter can also be understood as an example of an online community for participants. Using Baym's (2015) five features of online community helps to explain this. 
Firstly, there is a shared sense of space where these interactions are occurring. Secondly, interactions are normally of the same purpose - to communicate with others. Thirdly, the platform allows its users the opportunities to offer advice and support to one another, which most participants did. Narratives around discrimination, such as hate speech, were absent and more supportive environment was discussed. Fourthly, participants had a sense of shared identity with those they interacted with, particularly as they mainly followed and were followed by other pups. Finally, the platform allowed interpersonal relationships to occur through the communication tools between two or more people.

The cultural visibility and openness which the pup community has on Twitter is markedly different to how early examples of kink communities and sexual minorities operated. Arguably, Twitter serves a similar role to the Molly Houses and gay bars described earlier - allowing a space for sexual minorities to interact with each other in a space relatively free from stigma and which places emphasis on subcultural membership. However, discretion and secrecy played a pivotal role in Molly Houses and the kink communities of the Catacombs documented by Rubin (1991), and featuring to a lesser extent in kink communities described by others (e.g. Newmahr 2011; Weiss 2011). While privacy and discretion on Twitter is negotiated by participants, there is recognition that Twitter is a semipublic platform in so much as participants can use privacy settings to navigate levels of disclosure online and interactions can be witnessed by multiple people.

There are two key explanations for the shift from a need for kink to be 'underground' to the formation of a community in an online semi-public platform. Firstly, young people who have grown up in the internet age consider engaging with the sexual online as a mundane component of sexual life (Waskul 2015). Indeed, this is reflective of how young people engage with technology more broadly - tending to post greater amounts of personal information than older people. Individuals with kink interests can use the internet to 
communicate with like-minded people, opening up the possibility for these online communities to form. While this can be done in a public way, such as on Twitter, or more privately through the use of profile based websites, there is still a proliferation in these interactions.

Secondly, significant social change related to sexuality has occurred over the past few decades. Sexual acts rendered shocking in previous years are no longer viewed in the same way. For example, pornography is starting to be seen as a leisure activity (McCormack and Wignall 2016; Paasonen 2007); attitudes towards non martial sex have liberalized (Twenge et al. 2015); attitudes toward gays and lesbians has significantly improved in the West (Anderson and McCormack 2014; McCormack, 2012); and the primary motive for sex is moving away from procreation to recreation and a focus on pleasure (Treas et al. 2014). As part of this cultural change, there is a very public discourse about kinky sex, emphasised with the release of 50 Shades of Grey (Deller and Smith 2013) and kinky sex may no longer be as taboo as it once was (c.f. Rubin 1981).

As a way of understanding changing attitudes and behaviours towards sex, Attwood and Smith (2013) conceptualise the term 'leisure sex.' They highlight the benefits of labelling sex as a leisure activity, akin to other leisure pursuits, such as sport. They argue sex has 'significant benefits (and costs) for individuals and society, offering considerable potential for productivity, development of skills and knowledge, and thereby might engender selfconfidence, identity and community through achievement' (Attwood and Smith 2013: 330). Recognising sex as a leisure activity allows for an alternative discourse to emerge - rather than using a medicalised framework to view sex in terms of the risks of the behaviours (Newmahr 2011), viewing sex as a leisure activity allows for a recognition of risk alongside the pleasures of the activity. This perspective also compliments critical analyses of the use of SNS in these cultures, with the focus away from risk and harm. 
Despite liberalisation of attitudes, participants' use of a semi-public platform alongside their concerns around their pup identities raises interesting questions around the public vs private debate. How can participants openly interact in these online spaces and negotiate their pup identities while trying to maintain a level of secrecy and privacy? This type of activity is reminiscent of early sexual minorities interacting on chat rooms and online forums, while trying to maintain a heterosexual identity offline around friends and family (Mowlabocus 2010). It may be that the perceived stigma felt by the participants is similar to the stigma attached to homosexuality in previous eras; if this is the case, then one hopes similar levels of tolerance and acceptance happens for kink as it has to homosexuality in Western cultures (Twenge et al. 2015).

While the data from this paper cannot be generalised to all sexual communities or subcultures, it provides an insight into how SNS, such as Twitter, are being adopted into serving alternative functions - namely the construction of online communities. Moreover, it provides an opportunity for further research to explore how other sexual subcultures, and indeed non-sexual ones, are adapting to both social and technological changes. It also contributes to debates about the interaction between technology and social communities, demonstrating that innovations in social practice and technology use occur simultaneously among this group (David 2010).

In conclusion, this article has provided an insight into how a sexual subculture has used Twitter - to the extent that the interactions between users seen to be involved in this online community can be labelled as operating as part of Pup Twitter. Twitter is allowing this subculture to flourish online, exploiting Twitter's functions to engage in online interactions and find others with similar interests, and offline, allowing offline interactions to continue in an online environment. While this research has provided insight in the intersection between 
sexual subcultures and social networking sites, further research still needs to be undertaken on the social aspects of this phenomenon. 


\section{References}

ANDERSON E and McCormack M (2014) The influence of declining homophobia on men's gender in the United States: An argument for the study of homohysteria. Sex Roles, Vol. 71, No. 3-4, p. 109-120.

ATTWOOD F and Smith C (2013) More sex! Better sex! Sex is fucking brilliant! Sex, sex, sex, SEX. In: Blackwood T (Eds.) Routledge Handbook of Leisure Studies. New York, NY: Routledge.

BLACKWELL C, Birnholtz J and Abbott C (2014) Seeing and being seen: Co-situation and impression formation using Grindr, a location-aware gay dating app. New Media \& Society, Vol. 17, No. 7, p. 1117-1136.

BAYM NK (2015) Personal Connections in the Digital Age. John Wiley \& Sons.

BAYM NK (2007) The new shape of online community. First Monday, Vol. 12, No. 8.

BOASE J and Wellman B (2006) Personal relationships. In: Vangelisti A, Perlman D (Eds). The Cambridge Handbook of Personal Relationships. London: Cambridge University Press.

BOYD D (2007) Why youth (heart) social network sites: The role of networked publics in teenage social life. In: Buckingham D (Eds.) MacArthur Foundation Series on Digital Learning. Cambridge, MA: MIT Press.

BOYD DM and Ellison NB (2007) Social Network Sites: Definition, History and Scholarship. Journal of Computer-Mediated Communication, Vol. 13, No. 1, p. 210230.

CALLANDER D, Holt M, and Newman CE (2016) 'Not everyone's gonna like me': Accounting for race and racism in sex and dating web services for gay and bisexual men. Ethnicities, Vol. 16, No. 1, p. 3-21.

CHARMAZ K (2014) Constructing Grounded Theory. London, England: Sage. 
CHAYKO M (2008) Portable Communities: The Social Dynamics of Online and Mobile Connectedness. SUNY Press.

DAVID M (2010) Peer to Peer and the Music Industry. London: Sage.

DE SOUZA CS and Preece J (2004) A framework for analyzing and understanding online communities. Interacting with Computers, Vol. 16, No. 3, p. 579-610.

DELLER RA and Smith C (2013) Reading the BDSM romance. Sexualities, Vol. 16, No. 8, p, 932-950.

DEY I (1993) Qualitative Data Analysis. London: Routledge.

DÖRING N (2009) The Internet's impact on sexuality: A critical review of 15 years of research. Computers in Human Behavior, Vol. 25, No. 5, p. 1089-1101.

DRESCHER J (2015) Out of the DSM: depathologizing homosexuality. Behavioral Science, Vol. 5, No. 4, p. 565-575.

ELLISON NB, Steinfield C and Lampe C (2007) The benefits of Facebook “friends". Journal of Computer-Mediated Communication, Vol. 12, No. 4, p. 1143-1168.

FRANK K (2013) Plays Well in Groups: A Journey Through the World of Group Sex. Lanham, MD: Rowman \& Littlefield.

GHAZIANI A (2014) There Goes the Gayborhood? Princeton University Press.

GRAY M (2009) Out in the Country. New York: New York University Press.

GRUZD A, Wellman B and Takhteyev Y (2011) Imagining Twitter as an imagined community. American Behavioral Scientist, Vol. 55, No. 10, p. 1294-1318.

GUDELUNAS D (2012) There's an app for that: The uses and gratifications of online social networks for gay men. Sexuality \& Culture, Vol. 16, No. 4, p. 347-365.

HARRINGTON S, Highfield T, and Bruns A. (2012) More than a backchannel: Twitter and television. Audience Interactivity and Participation, Vol. 10, No. 1, p. 13-17 HINES C (2000) Virtual Ethnography. London: Sage. 
HONEYCUTT C and Herring C (2009) Beyond microblogging: Conversation and collaboration via Twitter. Proceedings of the Forty-second Hawaii International Conference on System Sciences (HICSS-42). Los Alamitos, CA: IEEE Press

HU M, Liu S, Wei F, Wu Y, Stasko J and Ma K (2012) Breaking news on Twitter. Proceedings of the SIGCHI Conference on human Factors in computing Systems. 2751-2754. ACM.

JASPAL R (2016) Gay men's construction and management of identity on Grindr. Sexuality and Culture. Online first, p. 1-18.

KRAUT R, Patterson M, Lundmark V, Kiesler S, Mukophadhyay T, and Scherlis W (1998) Internet paradox: A social technology that reduces social involvement and psychological well-being? American Psychologist, Vol. 53, No. 9, p. 1017-1031.

MCCORMACK M and Wignall L (2016) Enjoyment, Exploration and Education:

Understanding the Consumption of Pornography among Young Men with NonExclusive Sexual Orientations. Sociology. Online first.

MCCORMACK M (2012) The Declining Significance of Homophobia. New York: Oxford University Press.

MONDIN A (2017) 'Tumblr mostly, great empowering imag': blogging, reblogging and scrolling feminist, queer and BDSM desires. Journal of Gender Studies. Online first, p. 0-11.

MOORHEAD S, Hazlett D, Harrison L, Carroll J, Irwin A and Hoving C (2013) A new dimension of health care. Journal of Medical Internet Research, Vol. 15, No. 4, p. e85.

MOWLABOCUS S (2010) Gaydar Culture. London: Ashgate.

MURTHY D (2010) Muslim punks online. South Asian Popular Culture, Vol. 8, No. 2, p. 181-194. 
MURTHY D (2012) Towards a sociological understanding of social media: Theorizing Twitter. Sociology, Vol. 46, No. 6, p. 1059-1073.

NEWMAHR S (2011) Playing on the Edge: Sadomasochism, Risk and Intimacy.

Bloomington, IN: Indiana University Press.

NEWMAHR S (2015) Sharing and Waiting on Facebook. In: Waskul D and Vannini P (Eds.) Popular Culture as Everyday Life. New York: NY. Routledge.

NORTON R (1992) Mother Clap's Molly House. GMP Publishers.

PAASONEN S, Nikunen K and Saarenmaa, L (2007) Pornification: Sex and Sexuality in Media Culture. London, England: Berg.

PEAKMAN J (2013) The Pleasure's All Mine: A History of Perverse Sex. Reaktion Books. London: England.

PLUMMER K (1995) Telling Sexual Stories. London. Routledge.

PREECE J and Maloney-Krichmar D (2005) Online communities: Design, theory, and practice. Journal of Computer-Mediated Communication, Vol. 10, No. 4.

REHOR J (2015) Sensual, erotic, and sexual behaviors of women from the "kink" community. Archives of Sexual Behavior, Vol. 44, No. 4, p. 825-836.

RUBIN G (1981) The leather menace: Comments on politics and S/M. In: Samois (Eds.) Coming to Power. San Francisco: CA.

RUBIN G (1991) The catacombs: A temple of the butthole. In Thompson M (Eds.) Leatherfold: Radical sex, People, Politics and Practice. Boston, MA: Alyson.

SMITH MA and Kollock P (1999) Communities in Cyberspace. London: Routledge.

SOH D and Cantor J (2015) A peek inside a furry convention. Archives of Sexual Behavior, Vol. 44, No. 1, p. 1-2.

TREAS J, Lui J and Gubernskaya Z (2014) Attitudes on marriage and new relationships. Demographic Research, Vol. 30, No. 54, p. 1495-1526. 
TIIDENBERG K (2016) Boundaries and conflict in a NSFW community on tumblr: The meanings and uses of selfies. New Media and Society, Vol. 18, No. 8, p. 1563-1578. TURKLE S (2011) Alone Together. New York, NY: Basic Books.

TWENGE JM, Sherman RA and Wells BE (2015) Changes in American adults' sexual behavior and attitudes, 1972-2012. Archives of Sexual Behavior, Vol. 44, No. 8, p. $2273-2285$.

URQUHART C (2013) Grounded Theory for Qualitative Research. London, England: Sage. VICE (2015) Young gays are sniffing out the pup life. Vice. Available at: http://www.vice.com/en_uk/read/young-gays-are-sniffing-out-the-pup-life-979.

WAKEFORD N (2002) New technologies and 'cyberqueer' research. In: Richardson D and Sediman S (Eds.) Handbook of Lesbian and Gay Studies. London: Sage.

WASKUL D (2003) Self-games and body-play. Peter Lang: New York, NY.

WASKUL D (2015) Techno-sexuality: The sexual pragmatists of the technological age. In: Weinberg TS and Newmahr S (Eds.) Selves, Symbols and sexualities. London: Sage. WEEK J (2007) The World We Have Won. London: Routledge.

WEISS M (2011) Techniques of Pleasure. Durham, NC: Duke University Press.

WIGNALL L and McCormack M (2017) An exploratory study of a new kink activity: Pup play. Archives of Sexual Behavior, Vol. 46, No. 3, p. 801-811. 